\title{
Direct Observation of Metallic Thin Layers Dewetting By HT-ESEM
}

P. Jacquet ${ }^{1,2,3}$, B. Song ${ }^{2}$, R. Podor ${ }^{4}$, J. Lautru ${ }^{4}$, E. Ruiz Trejo ${ }^{5}$, S. J. Cooper ${ }^{6}$, J. Teisseire ${ }^{1}$, I. Gozhyk $^{1}$, J. Jupille ${ }^{2,3}$, R. Lazzari ${ }^{2,3}$

${ }^{1 .}$ Surface du Verre et Interfaces, UMR 125 CNRS/Saint-Gobain Recherche, 39 quai Lucien Lefranc, F-93303 Aubervilliers, France.

${ }^{2 .}$ CNRS, UMR 7588, Institut des NanoSciences de Paris, 4 place Jussieu, F-75005 Paris, France.

${ }^{3 .}$ Sorbonne Universités, UPMC Univ Paris 06, UMR 7588, Institut des NanoSciences de Paris, 4 place Jussieu, F-75005 Paris, France.

4. ICSM, CEA, CNRS, ENSCM, Univ Montpellier, Marcoule, France.

${ }^{5 .}$ Department of Earth Science and Engineering, Imperial College London, United Kingdom

6. Dyson School of Design Engineering, Imperial College London, London, SW7 1NA, UK.

During the last few years, an increasing research effort has been dedicated to the solid-state dewetting of metallic thin films [1]. It is identified as a potential way to produce at will metallic structures for numerous applications but it can also be an unexpected process for specific applications. However, for each application, the control of the morphology obtained through dewetting is crucial. In this respect, the understanding of the physical phenomena driving dewetting in polycrystalline films has been greatly improved. The role of grains has been underlined [2], new diffusion pathways have been identified and the role of crystalline orientation in anisotropic materials has been explored. In the present study, the dewetting of metallic thin layers will be investigated through in situ and real time HT-ESEM.

In the first example, solid-state dewetting of polycrystalline silver thin films was investigated in different annealing atmospheres: secondary vacuum or oxygen-rich (partial pressure $400 \mathrm{~Pa}$ ) environment (Fig. 1). A model where oxygen plays a key role is proposed to explain the very different observed morphologies; oxygen favors hole creation and isotropic hole propagation as well as grain selection. But, whatever the atmosphere, dewetting does not proceed through the propagation of a rim but instead involves the growth of specific grains and shrinkage of others [3].

In the second example, the isothermal dewetting behavior of $40 \mathrm{~nm} \mathrm{Ni}$ thin film on the yttriastabilized zirconia substrate have been investigated with a controlled gas mixture (Fig. 2). Indeed, during the fuel cell operation, the solid-state dewetting of the nickel leads to significant changes in electrode morphology that deteriorates its electrochemical performances. The material degradation was observed and quantified though the determination of Triple Phase Boundary (TPB) density. The coupling of morphological statistics and electrochemical performance degradation enabled qualitative discussion of the observed processes.

\section{References:}

[1] F Leroy et al, Surf Sci Rep 71 (2016), p. 391.

[2] P Jacquet et al, Scr Mater 115 (2016), p. 128.

[4] P Jacquet et al, Acta Materialia 143 (2018), p. 281. 

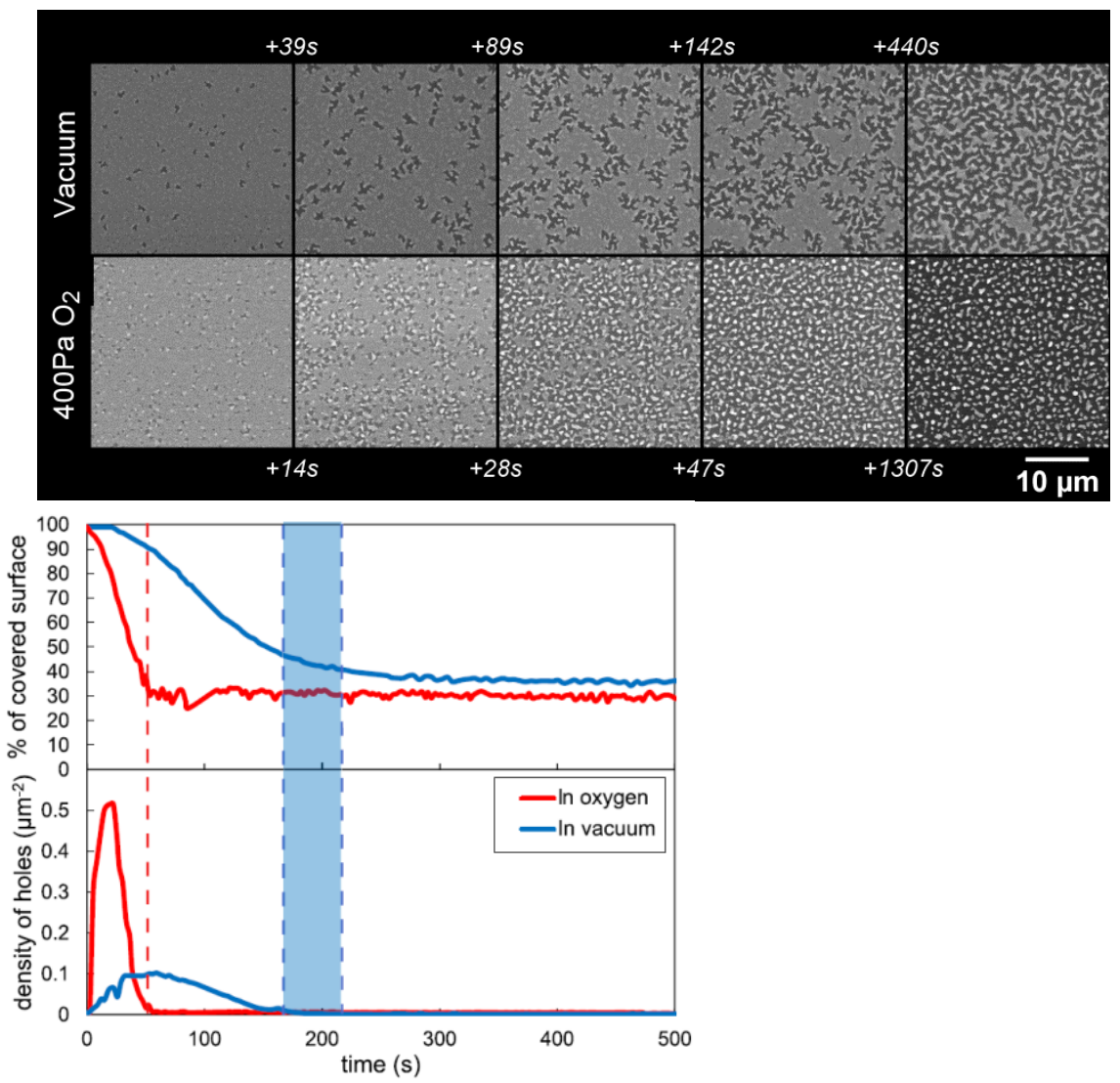

Figure 1. (a) Snapshots of a $40 \mathrm{~nm}$ silver layer dewetting in vacuum and $400 \mathrm{~Pa} \mathrm{O}_{2}$ at $350{ }^{\circ} \mathrm{C}$. (b) Data extracted from image series

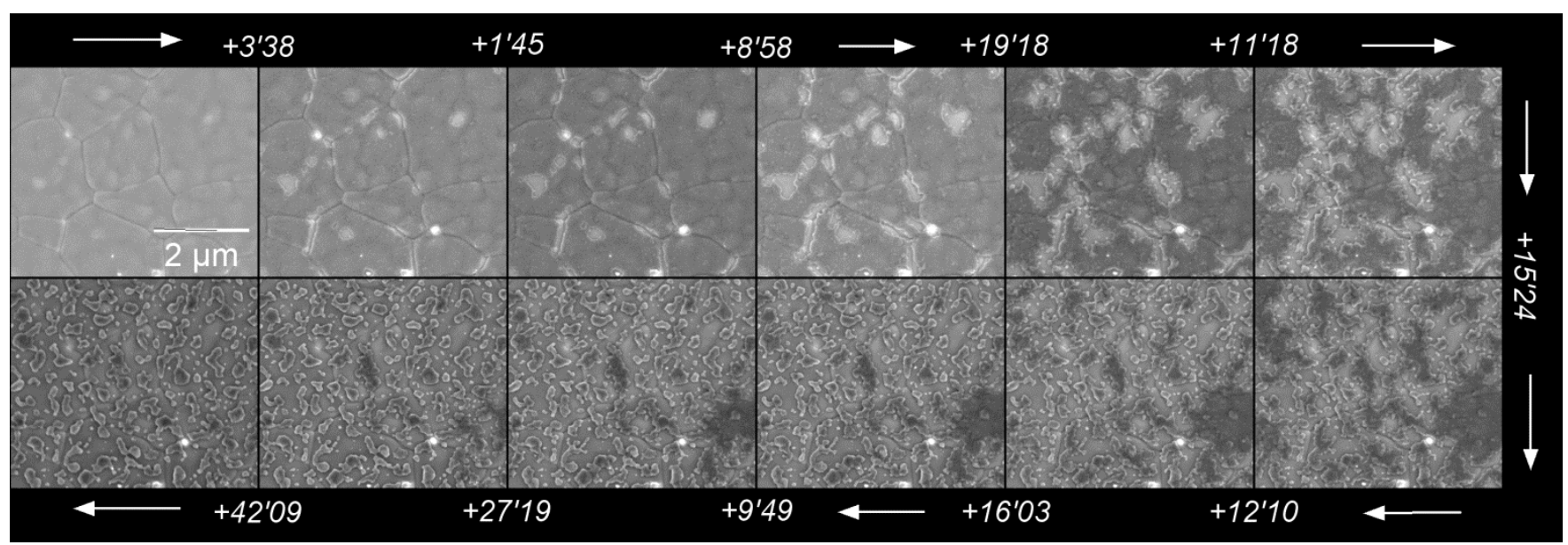

Figure 2. Snapshots of a $40 \mathrm{~nm}$ silver layer dewetting in the controlled gas mixture at $575^{\circ} \mathrm{C}$. The direction of the arrows indicates increasing time scale. 